\title{
Bubbling in Unbounded Coflowing Liquids
}

\author{
Alfonso M. Gañán-Calvo, ${ }^{1, *}$ Miguel A. Herrada, ${ }^{1, \dagger}$ and Piotr Garstecki ${ }^{2, \dagger}$ \\ ${ }^{1}$ Escuela Superior de Ingenieros, Universidad de Sevilla, Camino de los Descubrimientos s/n, 41092 Sevilla, Spain \\ ${ }^{2}$ Institute of Physical Chemistry, Polish Academy of Sciences, Kasprzaka 44/52, 01-224 Warsaw, Poland
}

(Received 19 September 2005; published 30 March 2006)

An investigation of the stability of low density and viscosity fluid jets and spouts in unbounded coflowing liquids is presented. A full parametrical analysis from low to high Weber and Reynolds numbers shows that the presence of any fluid of finite density and viscosity inside the hollow jet elicits a transition from an absolute to a convective instability at a finite value of the Weber number, for any value of the Reynolds number. Below that critical value of the Weber number, the absolute character of the instability leads to local breakup, and consequently to local bubbling. Experimental data support our model.

Bubbles have always stimulated scientific curiosity [e.g., [1] and references therein], but now microbubbles have become a hot research icon [e.g., [2-8], and references therein] for their increasing number of extraordinary applications in the fields of biomedicine [e.g., in diagnosis, as potential gene therapy vectors [2], to convey tiny amounts of therapeutic gases in the bloodstream without the risk of embolism, etc.], advanced physics studies [bubble sonoluminiscence [9], damping agents in neutron spallation sources, etc.], chemical engineering, and as a strong allied in environmental protection [e.g., microbubble drag reduction $[3,10]$ in marine transport, dissolved air flotation water depuration techniques, etc.].

The formation of micron-sized bubbles in a coflowing liquid-gas stream forced through minute orifices and microfluidic contractions has been the subject of previous experimental and theoretical studies [4-7,11]. In [6], the authors showed that depending on the Weber or Reynolds numbers range, monodisperse, bidisperse, or polydisperse microbubbling may occur. Besides, in the low Reynolds regime, both analytical [12] and experimental [13] studies support the existence of steady solutions for the formation of extremely thin and long fluid threads in a viscous coflowing liquid flow. Yet, fundamental questions remain unanswered: how stable is a long fluid filament in a coflowing liquid for any combination of densities, viscosities, surface tension, and coflow velocity? Is there a bubbling/ jetting transition like in liquid jets [e.g., [14]]?

To address these questions, we studied the convective or absolute instability of capillary jets in a coflowing unbounded heavier and more viscous liquid medium, over a wide range (6 orders of magnitude) from low to high Reynolds ( $\mathrm{Re}$ ) and Weber (We) numbers. In contrast to what occurs to liquid jets [15], we found that hollow jets are absolutely unstable, leading to local bubbling for all values of the Reynolds and Weber numbers of the coflowing liquid. However, the presence of any other fluid with finite viscosity and density inside the jet elicits a transition of the instability from absolute to convective for a finite
$\{\mathrm{Re}, \mathrm{We}\}$ pair. This transition corresponds to the bubblingjetting crisis similar to the dripping-jetting transition for capillary liquid jets [14]. Experiments support our findings, which delimit for the first time the wide parametrical realm of microbubbling in unbounded coflowing liquids.

Theoretical model and dispersion relation. - We studied the spatial-temporal response to small perturbations of an infinite cylindrical jet of an incompressible fluid (density $\rho_{g}$ and viscosity $\mu_{g}$ ) with radius $R_{j}$ in an immiscible unbounded liquid of larger density $\rho_{l}$ and viscosity $\mu_{l}$, such that $\alpha=\rho_{l} / \rho_{g}>10$ and $\beta=\mu_{l} / \mu_{g}>10 . \sigma$ is the surface tension between both fluids, which move with a uniform velocity $U$ relative to the observer in the $z$ direction. Reynolds and Weber numbers are defined as $\mathrm{Re}=$ $\rho_{l} U R_{j} / \mu_{l}$ and $\mathrm{We}=\rho_{l} U^{2} R_{j} / \sigma$, respectively. We made the small perturbations proportional to $e^{i(k z-\omega t)}$, where wave frequency $\omega$, time $t$, wave number $k$, and streamwise coordinate $z$ were made dimensionless with $U / R_{j}, R_{j} / U$, $1 / R_{j}$, and $R_{j}$, respectively. The general reader might go directly to the theoretical results section.

The full conservation equations of mass and momentum of the incompressible fluids flow relative to the observer [see, for example, [15] ], together with the boundary conditions at the jet surface (including normal and tangential stress balance) and at infinity, lead to the following dispersion relation between the perturbation wavelength $\omega$ and its wave number $k$ :

$$
\left(k^{2}-k_{g}^{2}\right)\left[\frac{N\left(k, k_{g}, k_{l}, \beta\right)}{M\left(k, k_{g}, k_{l}, \beta\right)}+2(1-\beta)\right]=\frac{\operatorname{Re}^{2}}{\mathrm{We}}\left(1-k^{2}\right),
$$

where "viscous" wave numbers are defined for both inner fluid and outer liquid as

$$
k_{g}^{2}=k^{2}-i \operatorname{Re} \hat{\omega}, \quad k_{l}^{2}=k^{2}-i \alpha \beta^{-1} \operatorname{Re} \hat{\omega},
$$

and functions $N$ and $M$ are expressed as 


$$
\begin{aligned}
N \equiv & 2 x \beta k_{g} k_{l}\left[K_{0}\left(k_{l}\right) I_{1}\left(k_{g}\right) k_{g}+I_{0}\left(k_{g}\right) K_{1}\left(k_{l}\right) k_{l}\right]+x\left[k^{2}(\beta-1)-k_{g}^{2}+\beta k_{l}^{2}\right]^{2} I_{0}(k) I_{1}\left(k_{g}\right) K_{0}(k) K_{1}\left(k_{l}\right) \\
& +4 k^{3} k_{g} k_{l}(\beta-1)^{2} I_{0}\left(k_{g}\right) I_{1}(k) K_{0}\left(k_{l}\right) K_{1}(k)-k_{l} I_{1}\left(k_{g}\right) K_{0}\left(k_{l}\right)\left\{\left[k^{4}+k_{g}^{2} k_{l}^{2}+k^{2}\left(k_{g}^{2}-k_{l}^{2}\right)\right] \beta I_{1}(k) K_{0}(k)\right. \\
& \left.+\left[k_{g}^{4}+k^{4}(1-2 \beta)^{2}-2 k^{2} k_{g}^{2}(\beta-1)\right] I_{0}(k) K_{1}(k)\right\}+k_{g} I_{0}\left(k_{g}\right) K_{1}\left(k_{l}\right)\left\{\left[k^{4}(\beta-2)^{2}+2 k^{2} k_{l}^{2} \beta(\beta-1)\right.\right. \\
& \left.\left.+\beta^{2} k_{l}^{4}\right] I_{1}(k) K_{0}(k)+\left[k^{2}\left(k^{2}-k_{g}^{2}\right)+k_{l}^{2}\left(k^{2}+k_{g}^{2}\right)\right] \beta I_{0}(k) K_{1}(k)\right\} \\
M \equiv x\left\{\left[k_{l}\right.\right. & \left.\left.K_{0}\left(k_{l}\right) K_{1}(k)-x K_{0}(k) K_{1}\left(k_{l}\right)\right]\left(k_{g}^{2}-k^{2}\right) I_{1}(k) I_{1}\left(k_{g}\right)+\beta\left[k_{g} I_{0}\left(k_{g}\right) I_{1}(k)-x I_{0}(k) I_{1}\left(k_{g}\right)\right]\left(k_{l}^{2}-k^{2}\right) K_{1}(k) K_{1}\left(k_{l}\right)\right\} .
\end{aligned}
$$

$I_{0}, I_{1}, K_{0}$, and $K_{1}$ stand for the modified Bessel functions of order 0 and 1. Funada and Joseph [16] obtained an equivalent expression for a temporal analysis. Here, consistently with a spatial-temporal stability analysis, we considered both $k$ and $\omega$ to be complex variables. Following the wellestablished spatial-temporal formalism [e.g., [17-21]] to describe the absolute or convective character of axisymmetric instabilities in the $\{\mathrm{Re}, \mathrm{We}, \alpha, \beta\}$ parametrical space of our problem, we sought solutions of $d \omega / d k=0$ in our dispersion relation (1) with nonzero imaginary parts of $\omega$ and $k$. We define the system to be absolutely unstable if there is at least one solution of the dispersion equation satisfying $d \omega / d k=0$ (zero group velocity) with $\operatorname{Im}(k)<$ 0 , and $\operatorname{Im}(\omega)>0$. Conversely, the system is convectively unstable if all possible solutions of the dispersion equation with zero group velocity have $\operatorname{Im}(k)<0$, and $\operatorname{Im}(\omega)<0$. We applied special care to choose all solutions whose spatial branches departing from the saddle point $d \omega / d k=$ 0 originate from distinct halves of the $k$ plane [[20], p. 484], i.e., the only ones providing the absolute instability growth rate.

We also used a spectral numerical code developed for our problem. This code is a based on that developed in [22] for the stability analysis of swirling flows in pipes. Here, the linearized equations were discretized in the $r$ direction using Chebyshev spectral collocation points $\left(n_{i}\right.$ points for the inner fluid and $n_{e}$ points for the outer one). For a given frequency $\omega$, we linearized the nonlinear (quadratic) eigenvalue problem for the wave number $k$ using the linear companion matrix method described in [23]. We solved numerically the resulting linear eigenvalue problem with the help of an eigenvalue solver subroutine (DGVCCG from the IMSL library) which provides the entire spectrum of eigenvalues and eigenfunctions. Spurious eigenvalues were ruled out by comparing the computed spectra obtained for different values of the number of collocation points. The use of the numerical procedures allowed us: (i) to check that both analytical and numerical techniques render the same results, and (ii) to use the numerical spectral technique in further studies to investigate the influence of other effects not considered here, such as the existence of other basic velocity profiles.

Theoretical results. - The causal link of the bubblingjetting transition with the onset of absolute instability is already well established [see, for example, [21] and references therein on this particular end]. Jetting occurs when the jet is convectively unstable to all wave solutions, while bubbling is elicited by the absolutely unstable response to at least one wave solution, whose amplitude grows locally without bounds. Here, for the sake of brevity, we only report the solution for each combination of $\{\mathrm{Re}, \mathrm{We}, \alpha, \beta\}$ with the largest temporal growth rate $\operatorname{Im}(\omega)>0$, i.e., the one that dominates the local breakup dynamics. In Fig. 1 we plotted the loci in the $\{\mathrm{Re}, \mathrm{We}\}$ plane for which $\operatorname{Im}(\omega)$ changes its sign. The line formed by these points mark the transition from a convective to an absolute $(C / A)$ character of the instability for a given set of values of $\{\alpha, \beta\}$.

Circles in Fig. 1 show the critical We numbers found with the numerical code for $\alpha=\beta=1000$ (we used $n_{i}=$ 31 and $n_{e}=71$ collocations points). There is a close agreement with the results obtained by solving the analytical dispersion relation (1). From the results above, given $\{\alpha, \beta\}$, one obtains that for small $\operatorname{Re}$ the $C / A$ transition depends on the capillary number $\mathrm{Ca}=\mathrm{We} / \mathrm{Re}$ only. For example, for $\alpha=\beta$, this results in an expression for the critical capillary number as $\mathrm{Ca}^{*}=0.139 \alpha^{1 / 2}$ for $\alpha$ above 100 . Below this critical capillary number, the instability is absolute (bubbling).

In the asymptotic limit of vanishing viscosity and density of the inner fluid $(\alpha, \beta \rightarrow \infty)$, the jet is absolutely unstable for any combination of values of the Reynolds and Weber numbers, which means that bubbling will be the norm for any finite Re and We. Alternatively, when the viscosity and density of the inner fluid are nonzero, for any finite value of the Reynolds number there is a finite value of

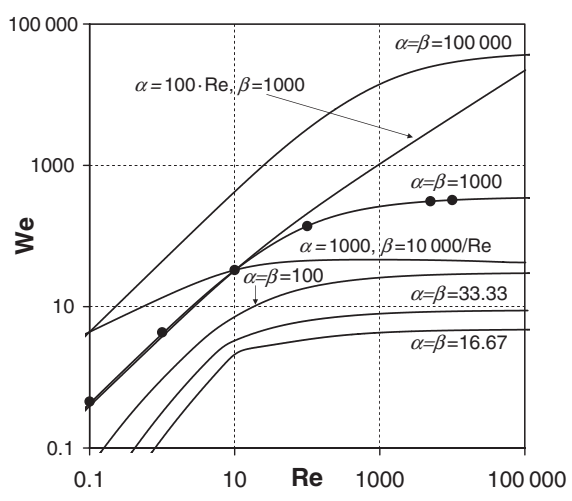

FIG. 1. Absolute or convective instability transition loci in the $\{\mathrm{Re}, \mathrm{We}\}$ plane for different values of $\{\alpha, \beta\}$. Regions below (above) the curves correspond to absolutely (convectively) unstable configurations. The region of absolute (convective) instability corresponds to bubbling (jetting). 
the Weber number at which the transition from absolute to convective instability takes place. Analogies with other capillary phenomena involving convective or absolute instability can be found in the literature [see, for example, $[24,25]]$. From all these studies, one may conclude that the absolute instability sets in when two circumstances coexist: (i) the system is unstable to a range of perturbations wavelengths, a feature that both convective and absolute instabilities share, and (ii) the upstream propagation velocity of at least a certain perturbation waveform just overcomes the convective velocity downstream. Consequently, an increase of the Reynolds and Weber numbers would foster a transition from absolute to convective instability by favoring convective speed against capillary growth. Our theoretical results agree with these general conclusions, as long as the fluid surface supporting the waves does not enclose a void (e.g., a hollow jet, with no fluid inside), i.e., as long as the capillary jet is filled with any fluid, which is the physically realistic case. Like in other fundamental phenomena in fluid dynamics (e.g., high Reynolds flows versus potential flows), this raises a severe caution when drawing physical conclusions from asymptotic limits out of the full expressions (1)-(4).

Experimental testing. - Here we used the experiments reported in Refs. [7,26], where a gaseous jet was pulled by a stream of liquid flowing through a coaxial orifice of diameter $D$ (flow focusing technique). Both the liquid and the gas issued from a stagnant region upstream of the orifice, where pressure is kept constant and equal for both fluids. The undisturbed gaseous jet diameter is calculated under the assumption that the gas velocity inside the undisturbed gas jet does not depart significantly from that of the coflowing liquid, which yields $d_{j} \simeq\left[4 Q_{g} /(\pi U)\right]^{0.5}$. We expect this approximation to be more accurate for smaller jet radii. In fact, owing to the limited values of the inner flow Reynolds number (here, from 5 to 50 ), a viscosity dominated flow regime would be very soon reached inside any developing jet, if such existed. Since the external unperturbed pressure is constant in our model (and in the experiments, after the exit orifice), any excess (defect) from the plug flow coming from a negative (positive) pressure gradient would involve a downstream increase (decrease) of the jet radius, which immediately would flatten the velocity profile by continuity arguments.

Although the onset of convective instability is often blurred by unsteadiness and chaotic bubbling [e.g., [6] ], we have occasionally observed in experiments what seems a clear transition to jetting, as reported in Fig. 2. Note that given $\alpha$ and $\beta$, for Re below 100, $C / A$ transition depends on the capillary number only; interestingly, in the experiment of Fig. 2, capillary velocity $\sigma / \mu \sim 11.5 \mathrm{~m} \cdot \mathrm{s}^{-1}$ seems to be just overcome by convective speed $U \simeq$ $4 Q_{l} /\left(\pi D^{2}\right)=12.2 \mathrm{~m} \cdot \mathrm{s}^{-1}$ when jetting takes place [Figs. 2(b) and 2(c)], while the former is larger than the latter $\left(U=6.9 \mathrm{~m} \cdot \mathrm{s}^{-1}\right)$ in Fig. 2(a) (bubbling), which allows a range of capillary waves to propagate upstream.

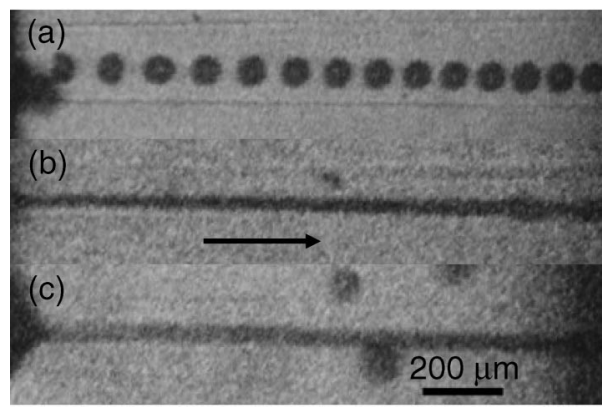

FIG. 2. Bubbling to jetting. Liquid: ethanol-water (50/50\% vv, $\left.\mu=0.002 \mathrm{~Pa} \cdot \mathrm{s}, \quad \sigma=0.023 \mathrm{~N} \cdot \mathrm{m}^{-1}\right), \quad T=28^{\circ} \mathrm{C}$. Orifice diameter $D=200 \mu \mathrm{m}$. (a) $Q_{l} \sim 13 \mathrm{~mL} / \mathrm{min}, \quad Q_{g} \sim$ $0.65 \mathrm{~mL} / \mathrm{min}$; (b) $Q_{l} \sim 23 \mathrm{~mL} / \mathrm{min}, Q_{g} \sim 0.25 \mathrm{~mL} / \mathrm{min}$; (c) $Q_{l} \sim 23 \mathrm{~mL} / \mathrm{min}, Q_{g} \sim 0.5 \mathrm{~mL} / \mathrm{min}$. The arrow indicates the flow direction; i.e., the nozzle is at the left of the photographs.

Although this is rather qualitative, it is consistent with our theoretical findings and physical causality.

In Fig. 3 we plotted the experimental microbubbling data points for three representative liquids $(\{\alpha=760, \beta=$ $68\},\{\alpha=800, \beta=324\}$, and $\{\alpha=850, \beta=544\}$ ) from Ref. [26], as well as the experiment of Fig. 2. Experimental points are located below the calculated Weber numbers for the transition from absolute to convective instability (i.e., they fall into the theoretical region of absolute instability). This is believed to support our model and to strengthen the causality linking absolute instability to bubbling.

It is worth emphasizing that the experimental issuing gas jets very presumably broke up before reaching the relaxed plug velocity profile in the bubbling regime. This fact does not invalidate the main conclusions from this study: on the contrary, for every bubbling experiment reported, if a gas

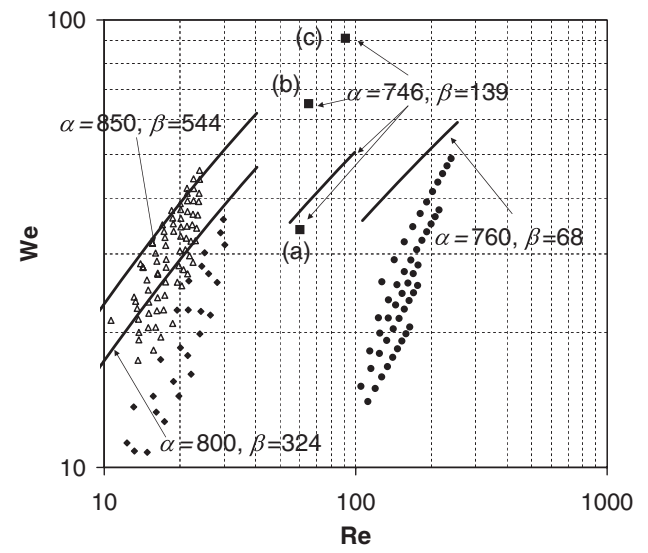

FIG. 3. Experimental values of $\{\mathrm{Re}, \mathrm{We}\}$ for four selected liquids (circles: $\{\alpha=760, \beta=68\}$; diamonds: $\{\alpha=800, \beta=$ 324\}; triangles: $\{\alpha=850, \beta=544\}$; squares: $\{\alpha=746, \beta=$ 139\}) from data in Ref. [26], and their corresponding $C / A$ instability transition curve under the assumption that $R_{j} \ll D$. The three square points (a), (b), and (c) correspond to the experiments given in Figs. 2(a)-2(c), respectively. 


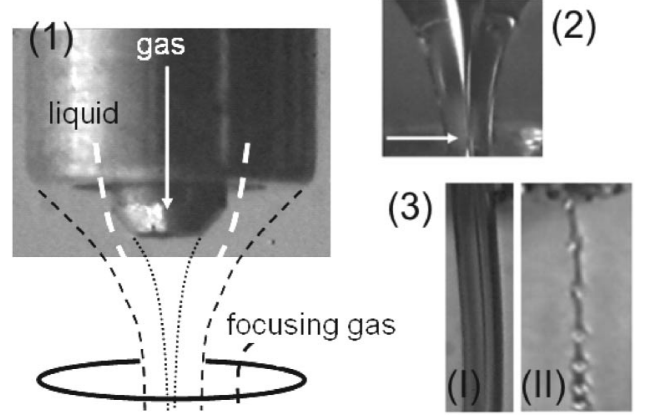

FIG. 4. (1) and (2) illustrative experimental configuration to produce a gas jet in an extensional viscous flow. A liquid (glucose syrup $\mu \simeq 7 \mathrm{~Pa} \cdot \mathrm{s}$ ) is forced to flow out of a capillary tube with $1 \mathrm{~mm}$ O.D. Inside it, a concentric tube with $0.4 \mathrm{~mm}$ O.D. feeds a continuous stream of gas. In this case, the liquid domain is not infinite since the extensional flow is produced by an external focusing gas forced through a $800 \mu \mathrm{m}$ orifice aligned with the concentric tubes at an offset adjustable distance from $0.5 \mathrm{~mm}$ to $1 \mathrm{~mm}$, as sketched [see Ref. [27]]. The orifice is bored in a $150 \mu \mathrm{m}$ thick plate. The gas pressure drop through the orifice is about $10 \mathrm{kPa}$. (3) A liquid jet with a gas core issuing from the orifice, as seen downstream. In (3)-(I) the jet is convectively unstable, while in (3)-(II) the jet "bubbles" right at the nozzle exit, indicating absolute instability.

jet would have developed, then it would have been absolutely unstable and never observed (i.e., local bubbling would be observed instead). This is fully consistent with results plotted in Fig. 3, where parametrical inputs in the absolute instability domain correspond to bubbling.

An important conclusion from this work is that a cylindrical gas spout in a high viscosity liquid moving with speed $U$ is absolutely unstable, preventing the formation of long gas spouts, below a finite value of the Weber number or, alternatively, below a finite value of the capillary number $\mathrm{Ca}=\mathrm{We} / \mathrm{Re}$. Thus, the elegant steady solution found by Zhang [12] for gas spouts entrained in extensional viscous flows would exist as long as the outer liquid is sufficiently viscous, provided that there is a minimum finite capillary number for which the steady solution can exist [12]. For $\operatorname{Re} \ll 1$, below the critical value of the capillary number, the spouts formed by the extensional flow in her solution would form a train of microbubbles, a fact observed in related experiments (see Fig. 4). For example, a hollow optical fiber with a cylindrical hole can only be drawn if the Weber number is above the critical one, or if there is an alternative mechanism providing local stability. This mechanism may be a longitudinal positive gradient of viscosity (e.g., the one due to "fiber quenching," when the glass solidifies) or an external negative pressure gradient [a potential alternative in ultrahigh speed fiber drawing, see Ref. [27] ].

This work is supported by the Ministry of Education and Science of Spain, Grants No. DPI2002-12345-C02 and No. DPI2004-07197, and by Ingeniatrics Technologies S. L.; P.G. acknowledges financial support from the
Foundation for Polish Science. Comments and corrections from Dr. P. Riesco-Chueca are also greatly appreciated. The experiment of Fig. 2 was performed by Alfonso Casado, Miguel Torres, and A.M. G. C.

*Email address: amgc@us.es

†Email address: herrada@us.es

\#Email address: garst@ichf.edu.pl

[1] A. Prosperetti, Phys. Fluids 16, 1852 (2004).

[2] C. D. Ohl and R. Ikink, Phys. Rev. Lett. 90, 214502 (2003).

[3] V.S. Lvov, A. Pomyalov, I. Procaccia, and V. Tiberkevich, Phys. Rev. Lett. 94, 174502 (2005).

[4] P. Garstecki, I. Gitlin, W. Diluzio, E. Kumacheva, H. A. Stone, and G. M. Whitesides, Appl. Phys. Lett. 85, 2649 (2004).

[5] P. Garstecki, H. A. Stone, and G. M. Whitesides, Phys. Rev. Lett. 94, 164501 (2005).

[6] P. Garstecki, M. J. Fuerstman, and G. M. Whitesides, Phys. Rev. Lett. 94, 234502 (2005).

[7] A. M. Gañán-Calvo and J. M. Gordillo, Phys. Rev. Lett. 87, 274501 (2001).

[8] P. Garstecki, A. M. Ganan-Calvo, and G. M. Whitesides, Bull. Pol. Acad. Sci. Tech. Sci. 53, 361 (2005).

[9] M. P. Brenner, S. Hilgenfeldt, and D. Lohse, Rev. Mod. Phys. 74, 425 (2002).

[10] A. Ferrante and S. Elghobashi, J. Fluid Mech. 503, 345 (2004).

[11] Y. Dimakopoulos and J. Tsamopoulos, Phys. Fluids 15, 1973 (2003).

[12] W. W. Zhang, Phys. Rev. Lett. 93, 184502 (2004).

[13] I. Cohen, H. Li, J. L. Hougland, M. Mrksich, and S. R. Nagel, Science 292, 265 (2001).

[14] P. Coullet, L. Mahadevan, and C. S. Riera, J. Fluid Mech. 526, 1 (2005).

[15] S. J. Leib and M. E. Goldstein, Phys. Fluids 29952 (1986).

[16] T. Funada, D. D. Joseph, and S. Yamashita, Int. J. Multiphase Flow 30, 1279 (2004).

[17] R. J. Briggs, Electron-Stream Interaction with Plasmas (MIT Press, Cambridge, MA, 1961).

[18] J. B. Keller, S. L. Rubinov, and Y. O. Tu, Phys. Fluids 16, 2052 (1973).

[19] S. J. Leib and M.E. Goldstein, J. Fluid Mech. 168, 479 (1986).

[20] P. Huerre and P. A. Monkewitz, Annu. Rev. Fluid Mech. 22, 473 (1990).

[21] J. M. Chomaz, Annu. Rev. Fluid Mech. 37, 357 (2005).

[22] M. R. Khorrami, M. R. Malik, and R. L. Ash, J. Comput. Phys. 81, 206 (1989).

[23] T. J. Bridges and P. J. Morris, J. Comput. Phys. 55, 437 (1984).

[24] M. Cheng and H.-C. Chang, Phys. Fluids 7, 34 (1995).

[25] H.-C. Chang, E. A. Demekhin, and D. I. Kopelevich, Phys. Rev. Lett. 75, 1747 (1995).

[26] A. M. Gañán-Calvo, Phys. Rev. E 69, 027301 (2004).

[27] A. M. Gañán-Calvo, M. Pérez-Saborid, J. M. LópezHerrera, and J. M. Gordillo, Eur. Phys. J. B 39, 131 (2004). 